\title{
Formation Control of Multiple Agents with Preserving Connectivity and its Application to Gradient Climbing
}

\author{
K.D. Do
}

\author{
K.D. Do \\ Department of Mechanical Engineering, \\ Curtin University of Technology, \\ Perth, WA 6845, Australia \\ E-mail: duc@curtin.edu.au
}

\begin{abstract}
A design of cooperative controllers that force a group of $N$ mobile agents with limited communication ranges to perform a desired formation is presented. The proposed formation control system also preserves initial communication connectivity and guarantees no collisions between the agents. The formation control design is based on smooth step functions, potential functions, and the Lyapunov direct method. The proposed formation control system is applied to solve a gradient climbing problem where the gradient average of a distributed field is estimated over a bounded region using the field measurement by the agents.

Keywords: Formation control, collision avoidance, gradient climbing.
\end{abstract}

\section{Introduction}

Formation control involves controlling positions of a group of agents such that they perform desired tasks such as optimizing objective functions from measurements taken by each agent, and stabilization/tracking desired locations relative to reference point(s). Various methods have been proposed for formation control of multiple agents.

Here, three popular methods are briefly mentioned. The leader-follower method (e.g., [1], [2]) uses several agents as leaders and others as followers. This method is easy to understand and ensures formation maintenance if the leaders are disturbed. However, the desired formation cannot be maintained if followers are perturbed unless a formation feedback is implemented, [3]. The behavioral method (e.g., [4], [5]), where each agent locally reacts to actions of its neighbors, is suitable for decentralized control but is difficult in control design and stability analysis since group behavior cannot explicitly be defined. The virtual structure method (e.g., [6], [7]) treats all agents as a single entity. This method is amenable to mathematical analysis but is difficult to deal with time-varying formation structure. Research works on formation control usually utilize one or more of the above methods in a centralized or a decentralized manner. Centralized strategies (e.g., [8], [3]) use a single controller that generates collision free trajectories in the workspace. These strategies guarantee a complete solution but require high computational power and are not robust. Decentralized schemes (e.g., [9], [10], [7]) require less computational effort but have difficulties in controlling critical points, especially when collision avoidance between the agents is a must.

The control design in the above works did not put hard constraints on the controlled outputs except for those papers considered the problem of collision avoidance. Without hard constraints on controlled outputs, overshoot might result in loss of initial communication between agents due to limited communication between the agents. Hard constraints on the controlled outputs were applied to design cooperative controllers for mobile agents to preserve initial communication. These constraints on the controlled outputs were obtained through barrier Lyapunov or potential functions using non-trivial bump functions or switching control strategies in [11] for the agreement problem, [12] for the centralized approach, and [13] for the swarm aggregation. 
This paper contributes two main folds. The first one is a design of smooth and bounded cooperative controllers for a group of mobile agents to perform a desired formation task. The desired formation task includes collision avoidance and communication connectivity preservation between the agents, time-varying desired formation shape, and stabilization of the desired formation shape at any reference trajectories with bounded time derivatives. The second contribution is an algorithm for estimating gradient average of a distributed field over a region in two dimensional space. This algorithm uses only the field measurement on the boundary of a region, over which the gradient average is to be estimated. The two contributions are then combined to provide an effective gradient climbing system for a group of mobile agents by allowing the reference trajectory for each agent generated based on the gradient average.

\section{Preliminaries and Formation Control Objective}

\subsection{Smooth step function}

This section presents a construction of a smooth step function. The smooth step function is to be embedded into a potential function to avoid discontinuities in the control law due to the agents' communication limitation in solving collision avoidance and connectivity preserving problems.

Definition 1. A scalar function $h(x, a, b, c)$ is said to be a smooth step function if it possesses the following properties where $x \in \mathbb{R}, h \prime(x, a, b, c)=\frac{\partial h(x, a, b, c)}{\partial x}, h \prime \prime(x, a, b, c)=$ $\frac{\partial^{2} h(x, a, b, c)}{\partial x^{2}}, a$ and $b$ are constants such that $a<b$, and $c$ is a positive constant.

Lemma 2. Let the scalar function $h(x, a, b, c)$ be defined as

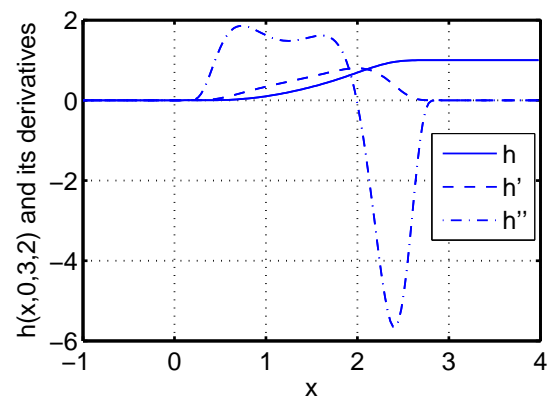

$$
h(x, a, b, c)=\frac{f(\tau)}{f(\tau)+c f(1-\tau)} \quad \text { with } \quad \tau=\frac{x-a}{b-a},
$$

(1) Figure 1: A smooth step function and its first and second derivatives.

where

$$
f(\tau)=0 \quad \text { if } \quad \tau \leq 0 \quad \text { and } \quad f(\tau)=e^{-\frac{1}{\tau}} \quad \text { if } \quad \tau>0,
$$

with $a$ and $b$ being constants such that $a<b$, and $c$ being a positive constant. Then the function $h(x, a, b, c)$ is a smooth step function.

Proof. Proof of this lemma follows the same lines as the proof of Lemma 1 in [7]. An illustration of a smooth step function $(a=0, b=3, c=2)$ is given in Figure 1. 


\subsection{Problem statement}

\section{Agent dynamics}

We assume that the agent $i$ has the dynamics:

$$
\dot{\boldsymbol{q}}_{i}=\boldsymbol{u}_{i}, i \in \mathbb{N},
$$

where $\mathbb{N}$ is the set of all agents in the group, $\boldsymbol{u}_{i} \in \mathbb{R}^{n}$ is the control input vector and $\boldsymbol{q}_{i} \in$ $\mathbb{R}^{n}$ the position vector of the agent $i$.

\section{Formation control objective}

Each agent in the group needs its reference trajectory to track. The reference trajectories can be predefined or determined from measurement data. Furthermore, each agent

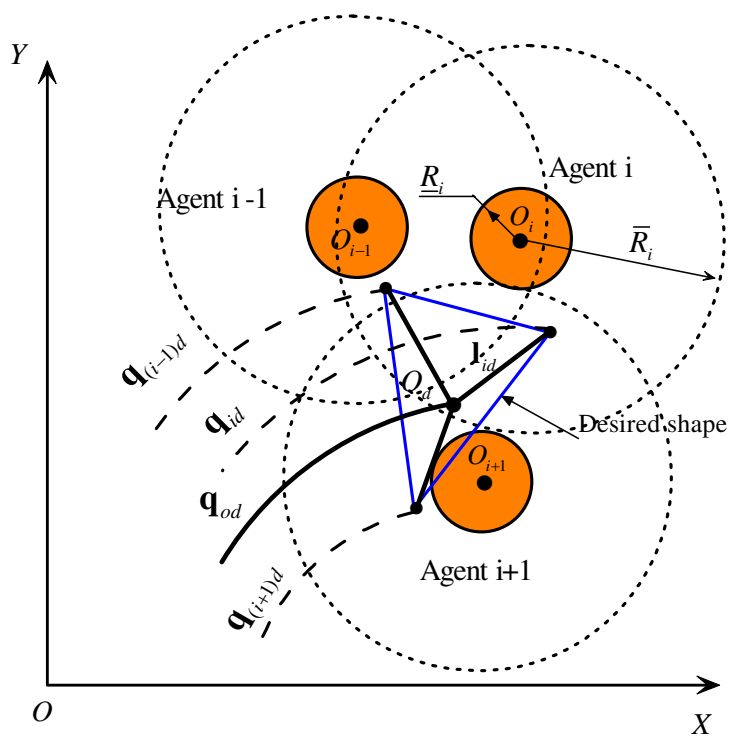

Figure 2: Formation setup. needs to communicate with other agents in the group to perform its cooperative mission. Therefore, before stating formation control objective we impose the following assumption on the reference trajectories, communication and initial conditions between the agents in the group:

\section{Assumption 3.}

1) The agent $i$ has a physical safety ball, which is centered at the point $O_{i}$ and has a radius $\underline{R}_{i}$, and has a communication ball, which is centered at the point $O_{i}$ and has a radius $\bar{R}_{i}$, see Figure 2. The radius $\bar{R}_{i}$ is such that

$$
\bar{R}_{i} \geq \underline{R}_{i}+\underline{R}_{j}+\varepsilon_{1 i j}
$$

for all $j \in \mathbb{N}, j \neq i$, where $\varepsilon_{1 i j}$ is a strictly positive constant.

2) The reference trajectory $\boldsymbol{q}_{i d}$ for the agent $i$ is generated by

$$
\boldsymbol{q}_{i d}=\boldsymbol{q}_{o d}\left(s_{o d}\right)+\boldsymbol{l}_{i d}
$$

where $\boldsymbol{q}_{o d}\left(s_{o d}\right)$ is referred to as the common reference trajectory with $s_{\text {od }}$ being the common trajectory parameter, and $\boldsymbol{l}_{i d}$ is to specify a desired formation shape. The trajectory $\boldsymbol{q}_{\text {od }}$ has its bounded derivatives. The vectors $\boldsymbol{l}_{\text {id }}, i \in \mathbb{N}$ have bounded derivatives, and satisfy

$$
\left(\underline{R}_{i}+\underline{R}_{j}+\varepsilon_{2 i j}\right) \leq\left\|\boldsymbol{l}_{i d}-\boldsymbol{l}_{j d}\right\| \leq \min \left(\bar{R}_{i}, \bar{R}_{j}\right)-\varepsilon_{2 i j},
$$

for all $(i, j) \in \mathbb{N}, i \neq j$, where $\varepsilon_{2 i j}$ is a strictly positive constant, and is strictly less than $\frac{\varepsilon_{1 i j}}{2}$.

3) The agent $i$ broadcasts its trajectory, $\boldsymbol{q}_{i}$, and its reference trajectory $\boldsymbol{q}_{i d}$ in its communication ball. Moreover, the agent $i$ can receive the trajectory, $\boldsymbol{q}_{j}$, broadcasted by other agents $j$, $j \in \mathbb{N}, j \neq i$ in the group if the points $O_{j}$ of these agents are in the communication ball of the agent $i$.

4) At the initial time $t_{0} \geq 0$, all the agents in the group are sufficiently far but not too far away from each other in the sense that the following condition holds:

$$
\left(\underline{R}_{i}+\underline{R}_{j}+\varepsilon_{3 i j}\right) \leq\left\|\boldsymbol{q}_{i}\left(t_{0}\right)-\boldsymbol{q}_{j}\left(t_{0}\right)\right\| \leq\left(\min \left(\bar{R}_{i}, \bar{R}_{j}\right)-\varepsilon_{3 i j}\right),
$$

for all $(i, j) \in \mathbb{N}, i \neq j$, where $\varepsilon_{3 i j}$ is a strictly positive constant and is strictly less than $\frac{\varepsilon_{1 i j}}{2}$. 
Remark 4. Item 2) in Assumption 3 defines a desired formation (by vectors $\boldsymbol{l}_{i d}$ ) and how this desired formation moves (by the common reference trajectory $\boldsymbol{q}_{o d}$ ). Item 3) specifies the way each agent communicates with other agents in the group within its communication range. In Figure 2, the agents $i$ and $i-1$ are communicating with each other since the points $O_{i-1}$ and $O_{i}$ are in the communication areas of the agents $i$ and $i-1$, respectively. Item 4) implies that at the initial time $t_{0}$ there are no collision between all the agents, and that all the agents are communicating with each other. The conditions (4), (6) and (7) are imposed to avoid conflict when solving collision avoidance and connectivity preserving problems. This is because we will design a formation control system so that $\boldsymbol{q}_{i}$ to track its reference trajectory $\boldsymbol{q}_{i d}$.

Under Assumption 3, for each agent $i$ design the control input vector $\boldsymbol{u}_{i}$ to achieve a desired formation consisting of 1) no switchings in the controllers; 2) no collisions between any agents; 3 ) asymptotic convergence of each agent's trajectory $\boldsymbol{q}_{i}$ to its reference trajectory $\boldsymbol{q}_{i d}$; and 4) initial connectivity preservation. Mathematically, the objective is to design a smooth $\boldsymbol{u}_{i}$ to achieve:

$$
\left\|\boldsymbol{q}_{i}(t)-\boldsymbol{q}_{j}(t)\right\|>\left(\underline{R}_{i}+\underline{R}_{j}\right), \lim _{t \rightarrow \infty}\left(\boldsymbol{q}_{i}(t)-\boldsymbol{q}_{i d}(t)\right)=0,\left\|\boldsymbol{q}_{i}(t)-\boldsymbol{q}_{j}(t)\right\|<\min \left(\bar{R}_{i}, \bar{R}_{j}\right),
$$

for all $\forall t \geq t_{0} \geq 0$ and $(i, j) \in \mathbb{N}$ and $j \neq i$.

\section{Formation Control Design}

Consider the following potential function

$$
\varphi=\sum_{i=1}^{N}\left(\gamma_{i}+\frac{1}{2} \beta_{i}\right)
$$

The aim of the goal function $\gamma_{i}$ is to achieve asymptotic convergence of each agent's trajectory $\boldsymbol{q}_{i}$ to its reference trajectory $\boldsymbol{q}_{i d}$. As such the function $\gamma_{i}$ puts penalty on the tracking errors between the trajectory $\boldsymbol{q}_{i}$ of the agent $i$ and its reference trajectory $\boldsymbol{q}_{i d}=\boldsymbol{q}_{o d}+\boldsymbol{l}_{i d}$. We choose the function $\gamma_{i}$ as:

$$
\gamma_{i}=\frac{1}{2}\left\|\boldsymbol{q}_{i}-\boldsymbol{q}_{i d}\right\|^{2}
$$

The purpose of the collision avoidance and connectivity preserving function $\beta_{i}$ is to force the agent $i$ to move away from other agents, and to maintain communication connectivity between the agent $i$ and other agents in the group. This function is chosen as follows:

$$
\beta_{i}=\sum_{j \in \mathbb{N}_{i}} \beta_{i j}
$$

where $\mathbb{N}_{i}$ is the set of all the agents in the group except for the agent $i$. The function $\beta_{i j}=\beta_{j i}$ is a function of $\left\|\boldsymbol{q}_{i j}\right\|^{2} / 2$ with $\boldsymbol{q}_{i j}=\boldsymbol{q}_{i}-\boldsymbol{q}_{j}$, and possesses the following properties:

1) $\beta_{i j}=0, \beta_{i j} \prime=0, \beta_{i j} \prime \prime=0, \forall\left\|\boldsymbol{q}_{i j}\right\| \in\left(\left(\underline{R}_{i}+\underline{R}_{j}+\delta_{i j}\right),\left(\min \left(\bar{R}_{i}, \bar{R}_{j}\right)-\delta_{i j}\right)\right)$,

2) $\beta_{i j}>0, \forall\left\|\boldsymbol{q}_{i j}\right\| \in\left(\left(\left(\underline{R}_{i}+\underline{R}_{j}\right),\left(\underline{R}_{i}+\underline{R}_{j}+\delta_{i j}\right)\right) \cup\left(\left(\min \left(\bar{R}_{i}, \bar{R}_{j}\right)-\delta_{i j}\right), \min \left(\bar{R}_{i}, \bar{R}_{j}\right)\right)\right)$,

3) $\quad \lim _{\left.\left\|\boldsymbol{q}_{i j}\right\| \rightarrow \underline{R}_{i}+\underline{R}_{j}\right)} \beta_{i j}=\infty, \lim _{\left\|\boldsymbol{q}_{i j}\right\| \rightarrow \min \left(\bar{R}_{i}, \bar{R}_{j}\right)} \beta_{i j}=\infty$,

4) $\beta_{i j}$ is smooth for all $\left\|\boldsymbol{q}_{i j}\right\| \in\left(\left(\underline{R}_{i}+\underline{R}_{j}\right),\left(\min \left(\bar{R}_{i}, \bar{R}_{j}\right)\right)\right)$, 
where $\delta_{i j}$ is a strictly positive constant and is strictly less than $\varepsilon_{2 i j}$ specified in Assumption 3 . The terms $\beta_{i j}$ and $\beta_{i j}$ ll are defined as follows:

$$
\begin{aligned}
& \beta_{i j}{ }^{\prime}=\infty, \beta_{i j} \prime \prime=\infty, \text { if }\left\|\boldsymbol{q}_{i j}\right\|=\underline{R}_{i}+\underline{R}_{j}, \text { or }\left\|\boldsymbol{q}_{i j}\right\|=\min \left(\bar{R}_{i}, \bar{R}_{j}\right), \\
& \beta_{i j^{\prime}}=\frac{\partial \beta_{i j}}{\partial\left(\left\|\boldsymbol{q}_{i j}\right\|^{2} / 2\right)}, \beta_{i j} \prime \prime=\frac{\partial^{2} \beta_{i j}}{\partial\left(\left\|\boldsymbol{q}_{i j}\right\|^{2} / 2\right)^{2}}, \text { elsewhere. }
\end{aligned}
$$

Based on the smooth step function in Section 2.1, we can find many functions that satisfy all properties listed in (12). As an example, we will use the following function $\beta_{i j}$ :

$$
\beta_{i j}=\kappa_{i j}\left[\frac{1-h\left(\frac{\left\|\boldsymbol{q}_{i j}\right\|^{2}}{2}, \frac{\left(\underline{R}_{i}+\underline{R}_{j}\right)^{2}}{2}, \frac{\left(\underline{R}_{i}+\underline{R}_{j}+\delta_{i j}\right)^{2}}{2}, c_{i j}\right)}{\left(\frac{\left\|\boldsymbol{q}_{i j}\right\|^{2}}{2}-\frac{\left(\underline{R}_{i}+\underline{R}_{j}\right)^{2}}{2}\right)^{2}}+\frac{h\left(\frac{\left\|\boldsymbol{q}_{i j}\right\|^{2}}{2}, \frac{\left(\min \left(\bar{R}_{i}, \bar{R}_{j}\right)-\delta_{i j}\right)^{2}}{2}, \frac{\min \left(\bar{R}_{i}, \bar{R}_{j}\right)^{2}}{2}, c_{i j}\right)}{\left(\frac{\min \left(\bar{R}_{i}, \bar{R}_{j}\right)^{2}}{2}-\frac{\left\|\boldsymbol{q}_{i j}\right\|^{2}}{2}\right)^{2}}\right],
$$

where $\kappa_{i j}$ and $c_{i j}$ are positive constants, and the function $h(\bullet)$ is a smooth step function defined in Definition 1. An illustration of $\beta_{i j}$ defined in (14) is given in Figure 3 with $\underline{R}_{i}+\underline{R}_{j}=1$, $\min \left(\bar{R}_{i}, \bar{R}_{j}\right)=11, \delta_{i j}=2, c_{i j}=1, \kappa_{i j}=1$.

The derivative of $\varphi$ along the solutions of (3) satisfies

$$
\dot{\varphi}=\sum_{i=1}^{N} \boldsymbol{\Omega}_{i}^{T}\left(\boldsymbol{u}_{i}-\dot{\boldsymbol{q}}_{i d}\right)+\sum_{i=1}^{N}\left(\sum_{j \in \mathbb{N}_{i}} \beta_{i j} \boldsymbol{q}_{i j}^{T}\right) \boldsymbol{i}_{i d},
$$

where

$$
\boldsymbol{\Omega}_{i}=\boldsymbol{q}_{i}-\boldsymbol{q}_{i d}+\sum_{j \in \mathbb{N}_{i}} \beta_{i j} / \boldsymbol{q}_{i j}
$$

From (15), we design the control input $\boldsymbol{u}_{i}$ to make the sum $\sum_{i=1}^{N} \boldsymbol{\Omega}_{i}^{T}\left(\boldsymbol{u}_{i}-\dot{\boldsymbol{q}}_{i d}\right)$ negative definite as

$$
\boldsymbol{u}_{i}=-k \boldsymbol{\Psi}\left(\boldsymbol{\Omega}_{i}\right)+\dot{\boldsymbol{q}}_{o d}+\dot{\boldsymbol{i}}_{i d},
$$

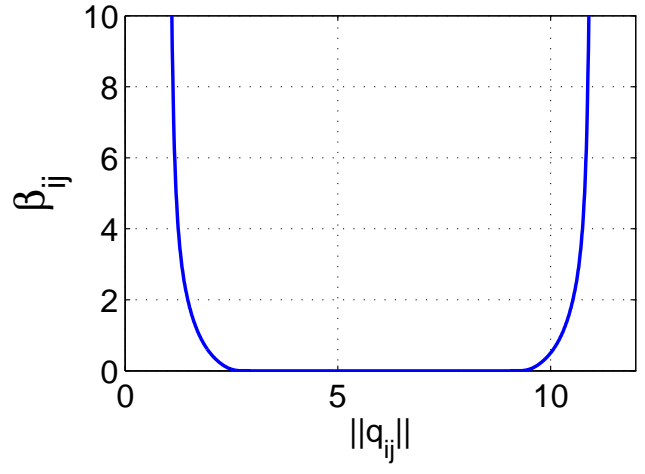

Figure 3: An illustration of $\beta_{i j}$.

where $k$ is a positive constant, and $\boldsymbol{\Psi}\left(\boldsymbol{\Omega}_{i}\right)$ denotes a vector of bounded functions of elements of $\boldsymbol{\Omega}_{i}$ in the sense that $\boldsymbol{\Psi}\left(\boldsymbol{\Omega}_{i}\right)=\left[\psi\left(\Omega_{i}^{1}\right) \ldots, \psi\left(\Omega_{i}^{l}\right), \ldots, \psi\left(\Omega_{i}^{n}\right)\right]^{T}$ with $\Omega_{i}^{l}$ the $l^{\text {th }}$ element of $\boldsymbol{\Omega}_{i}$, i.e., $\boldsymbol{\Omega}_{i}=\left[\Omega_{i}^{1} \ldots, \Omega_{i}^{l} \ldots \Omega_{i}^{n}\right]^{T}$. The function $\psi(x)$ satisfies

$$
\begin{aligned}
& \text { 1) }|\psi(x)| \leq M_{1} \text {, } \\
& \text { 2) } \psi(x)=0 \quad \text { if } x=0, \quad x \psi(x)>0 \text { if } x \neq 0 \text {, } \\
& \text { 3) } \psi(-x)=-\psi(x),(x-y)[\psi(x)-\psi(y)] \geq 0,4)\left|\frac{\psi(x)}{x}\right| \leq M_{2},\left|\frac{\partial \psi(x)}{\partial x}\right| \leq M_{3},\left.\frac{\partial \psi(x)}{\partial x}\right|_{x=0}=1 \text {, }
\end{aligned}
$$

for all $x \in \mathbb{R}, y \in \mathbb{R}$, where $M_{1}, M_{2}, M_{3}$ are strictly positive constants. Some functions that satisfy the above properties $\operatorname{are} \arctan (x)$ and $\tanh (x)$. The above bounds mean that the large control effort problem is avoided when the distance $\left\|\boldsymbol{q}_{i j}\right\|$ between the agent $i$ and an agent $j$ in the group reaches a collision limit $\underline{R}_{i}+\underline{R}_{j}$ or a connectivity preserving limit $\min \left(\bar{R}_{i}, \bar{R}_{j}\right)$.

To deal with the sum $\sum_{i=1}^{N}\left(\sum_{j \in \mathbb{N}_{i}} \beta_{i j} \boldsymbol{q}_{i j}^{T}\right) i_{i d}$ in (15), we observe that $\beta_{i j}$ ' $=0$ for all $\left\|\boldsymbol{q}_{i j}\right\| \in\left(\left(\underline{R}_{i}+\underline{R}_{j}+\delta_{i j}\right),\left(\min \left(\bar{R}_{i}, \bar{R}_{j}\right)-\delta_{i j}\right)\right)$, see Property 1$)$ of the function $\beta_{i j}$ in (12). This observation motivates us to design an update law for $\boldsymbol{l}_{i d}$ so that $\sum_{i=1}^{N}\left(\sum_{j \in \mathbb{N}_{i}} \beta_{i j} / \boldsymbol{q}_{i j}^{T}\right) \boldsymbol{i}_{i d}=0$ for all time and $\boldsymbol{l}_{i d}$ tends to its desired value $\boldsymbol{v}_{i d}$ asymptotically. As such, we choose:

$$
i_{i d}=H_{i} \boldsymbol{v}_{i d}
$$


where

$$
\begin{aligned}
H_{i}= & \prod_{j \in \mathbb{N}_{i}} h\left(\left\|\boldsymbol{q}_{i j}\right\|^{2} / 2,\left(\underline{R}_{i}+\underline{R}_{j}+\delta_{i j}\right)^{2} / 2,\left(\underline{R}_{i}+\underline{R}_{j}+\delta_{i j}^{v}\right)^{2} / 2, c_{i j}\right) \times \\
& \left(1-h\left(\left\|\boldsymbol{q}_{i j}\right\|^{2} / 2, \min \left(\bar{R}_{i}+\bar{R}_{j}-\delta_{i j}^{v}\right)^{2} / 2,\left(\bar{R}_{i}+\bar{R}_{j}-\delta_{i j}\right)^{2} / 2, c_{i j}\right)\right),
\end{aligned}
$$

with $\delta_{i j}^{v}$ being a positive constant such that $\delta_{i j}<\delta_{i j}^{v}<\epsilon_{2 i j}$, and $h(\bullet)$ being a smooth step function defined in Definition 1. With the choice of $\delta_{i j}<\delta_{i j}^{v}<\epsilon_{2 i j}$, we can see that

$$
\begin{aligned}
& H_{i}=1, \forall\left\|\boldsymbol{q}_{i j}\right\| \in\left(\left(\underline{R}_{i}+\underline{R}_{j}+\delta_{i j}^{v}\right),\left(\min \left(\bar{R}_{i}, \bar{R}_{j}\right)-\delta_{i j}^{v}\right)\right), \\
& \left.H_{i}=0, \forall\left\|\boldsymbol{q}_{i j}\right\| \in\left(\left(0,\left(\underline{R}_{i}+\underline{R}_{j}+\delta_{i j}\right)\right) \cup\left(\min \left(\bar{R}_{i}, \bar{R}_{j}\right)-\delta_{i j}\right), \infty\right)\right), \\
& 0<H_{i}<1, \text { elsewhere. }
\end{aligned}
$$

Obviously, the choice of the update law for $\boldsymbol{l}_{i d}$ in (19) with $H_{i}$ being satisfied (21) gives:

$$
\sum_{j \in \mathbb{N}_{i}} \beta_{i j} \boldsymbol{q}_{i j}^{T} \dot{i}_{i d}=0, \forall\left\|\boldsymbol{q}_{i j}\right\| \in\left(\left(\underline{R}_{i}+\underline{R}_{j}\right), \min \left(\bar{R}_{i}, \bar{R}_{j}\right)\right) .
$$

Remark 5. 1) A careful look at the control law $\boldsymbol{u}_{i}$ in (17) with $\boldsymbol{\Omega}_{i}$ in (16) shows that the argument of the bounded $\boldsymbol{\Psi}$ (with the negative sign moved in) consists of two parts. The first part is $-\left(\boldsymbol{q}_{i}-\boldsymbol{q}_{i d}\right)$, and the second part is $-\sum_{j \in \mathbb{N}_{i}} \beta_{i j} / \boldsymbol{q}_{i j}$. The first part together with $\dot{\boldsymbol{q}}_{o d}+\boldsymbol{i}_{i d}$ is referred to as the attractive force plays the role of forcing the agent $i$ to track its reference trajectory. The second part is referred to as the repulsive force takes care of collision avoidance and connectivity preserving for the agent $i$ with the other agents in the group. Moreover, the control $\boldsymbol{u}_{i}$ is a smooth function of and depend on only its own state and reference trajectory, and the states of other neighbor agents $j$ if the agents $j$ are sufficiently close to the agent $i$ for collision avoidance, or are sufficiently far away from the agent $i$ for connectivity preserving.

2) The choice of the update law in (19) ensures that when the collision avoidance or connectivity preserving is active, i.e., when the sum $\sum_{j \in \mathbb{N}_{i}} \beta_{i j} \boldsymbol{q}_{i j}$ is non-zero, the vector $\boldsymbol{l}_{i d}$ is not updated, i.e., the desired formation shape is not changed. This implies that the control law $\boldsymbol{u}_{i}$ gives priority to the collision avoidance and/or connectivity preserving mission or the desired formation shape updating mission whenever which mission is more important.

Substituting the control law $\boldsymbol{u}_{i}$ in (17) and the update law $\boldsymbol{i}_{i d}$ in (19) into (15) gives

$$
\dot{\varphi}=-k \sum_{i=1}^{N} \boldsymbol{\Omega}_{i}^{T} \boldsymbol{\Psi}\left(\boldsymbol{\Omega}_{\boldsymbol{i}}\right),
$$

where we have used (22). On the other hand, substituting the control law the control law $\boldsymbol{u}_{i}$ in (17) into (3) including the update law $\boldsymbol{l}_{i d}$ in (19) results in the closed loop system:

$$
\begin{aligned}
& \dot{\boldsymbol{q}}_{i}=-k \boldsymbol{\Psi}\left(\boldsymbol{\Omega}_{i}\right)+\dot{\boldsymbol{q}}_{o d}+\dot{\boldsymbol{i}}_{i d}, \\
& \dot{\boldsymbol{l}}_{i d}=H_{i} \boldsymbol{v}_{i d},
\end{aligned}
$$

for all $i \in \mathbb{N}$. We now present the main result of our paper in the following theorem.

Theorem 6. Under Assumption 3, the smooth control input $\boldsymbol{u}_{i}=$ given in (17) and the update law $\boldsymbol{i}_{i d}$ in (19) for the agent $i$ solve the formation control objective. In particular:

1) There are no collisions between any agents, connectivity between the agents is maintained, and the closed loop system (24) is forward complete. The first and last inequalities in (8) hold.

2) The reference velocity $\boldsymbol{i}_{i d}$ approaches its desired reference velocity $\boldsymbol{v}_{i d}$ asymptotically.

3) The trajectory $\boldsymbol{q}_{i}$ of each agent $i$ tracks its reference trajectory $\boldsymbol{q}_{i d}$ asymptotically, i.e., the limit in the second equation of (8) holds.

Proof. See Appendix 1. 


\section{Gradient climbing}

\subsection{Approach}

In this section, we present an application of our proposed formation control to solve a gradient climbing mission in a distributed environment $\Phi(t, \boldsymbol{\eta})$. To do so, we consider each agent in the group as a mobile sensor and the network as a reconfigurable sensor array. As such, at each time $t$ the agent $i$ with $i \in \mathbb{N}$ in the group of $N$ agents is equipped with a sensor that can measure $\Phi\left(t, \boldsymbol{q}_{i}\right)$ at the location $\boldsymbol{q}_{i}$. With $\Phi\left(t, \boldsymbol{q}_{i}\right)$, we estimate/calculate an approximation of the gradient average, $\bar{\nabla} \Phi$, of the distributed environment over a region $A$ bounded by a contour $C$, on which the agents in the group are positioned. After $\bar{\nabla} \Phi$ is estimated/calculated, we let the gradient of the common reference trajectory $\boldsymbol{q}_{o d}$ equal to $\bar{\nabla} \Phi$. This means that the common reference trajectory $\boldsymbol{q}_{o d}$ is simply generated by

$$
\dot{\boldsymbol{q}}_{\text {od }}=\frac{\partial \boldsymbol{q}_{o d}}{\partial s_{o d}} \dot{s}_{o d}=\bar{\nabla} \Phi \dot{s}_{o d}
$$

with some initial condition $\boldsymbol{q}_{o d}\left(t_{0}\right)$, where $\dot{s}_{\text {od }}$ specifies how fast the desired formation moves along the common reference trajectory $\boldsymbol{q}_{o d}$. For the case of gradient descent, we can use $\dot{\boldsymbol{q}}_{o d}=-\bar{\nabla} \Phi \dot{s}_{\text {od }}$ instead of (25). Moreover, we can specify the desired formation shape velocity $\boldsymbol{v}_{i d}$ to change (expand/shrink/rotate) the formation shape, i.e., change the shape define vector $\boldsymbol{l}_{i d}$, see (19), to improve the gradient average approximation. We propose the the desired formation shape velocity $\boldsymbol{v}_{i d}$ as follows:

$$
\boldsymbol{v}_{i d}=-\boldsymbol{K}_{1 v}\left(\boldsymbol{l}_{i d}-\boldsymbol{l}_{i d}^{*}\right)+\boldsymbol{K}_{2 v} \boldsymbol{\Psi}(\bar{\nabla} \Phi)
$$

where $\boldsymbol{K}_{1 v}$ and $\boldsymbol{K}_{2 v}$ are diagonal positive definite matrices. The constant vectors $\boldsymbol{l}_{i d}^{*}, i \in \mathbb{N}$ are chosen so that they specify the minimum desired formation shape, which is such that the condition (6) holds with $\boldsymbol{l}_{i d}$ replaced by $\boldsymbol{l}_{i d}^{*}$ for all $i \in \mathbb{N}$. The vector function $\boldsymbol{\Psi}(\bar{\nabla} \Phi)$ is a bounded vector function of $\bar{\nabla} \Phi$, see the paragraph just below (17). Once the common reference trajectory $\boldsymbol{q}_{o d}$ and the desired formation shape $\boldsymbol{l}_{i d}$ are available, the formation control design proposed in Section 3 can be used directly to drive the agents in the group. The following section gives a method to estimate an approximation of the gradient average, $\bar{\nabla} \Phi$, of the distributed environment $\Phi(t, \boldsymbol{\eta})$ from measurements $\Phi\left(t, \boldsymbol{q}_{i}\right)$ on the boundary, i.e., the contour or surface $C$, carried out by the agents in the group. Therefore, we will present a method to calculate the gradient average of a distributed field in the following subsection.

\subsection{Average gradient estimate of a distributed field}

We consider a region $A$, see Figure 4 , bounded by a contour $C$, such that any line through $A$ parallel to either one of the coordinate axes intersects $C$ in only two points. The curve $C$ is divided by its leftmost and rightmost points $(x=a$ and $x=b)$ into a lower segment $C_{1}$, described by $y=f_{1}(x)$, and an upper segment $C_{2}$ described by $y=$ $f_{2}(x)$. With the position vector to a point $P$ on $C$ given by $\boldsymbol{r}=x e_{x}+y e_{y}$, where $e_{x}$ and $e_{y}$ are the unit vector on the $O X$ and $O Y$ axes, respectively. The unit tangent vector at $P$ is $\boldsymbol{t}=\frac{d \boldsymbol{r}}{d s}=\frac{d x}{d s} e_{x}+\frac{d y}{d s} e_{y}$, where $d s$ is the differential length along $C$, and the unit normal vector is $\boldsymbol{n}=\boldsymbol{t} \times e_{z}=\frac{d y}{d s} e_{x}-\frac{d x}{d s} e_{y}=n_{x} e_{x}+n_{y} e_{y}$. For the function

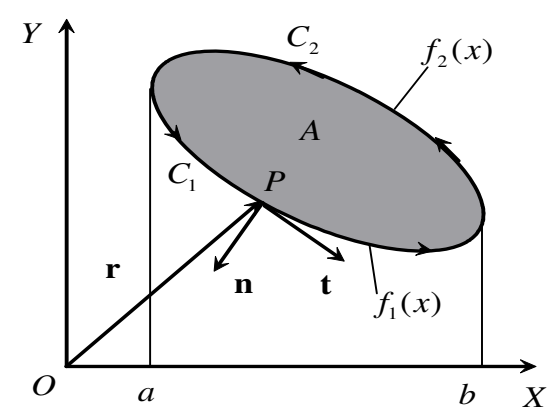

Figure 4: Coordinates for a gradient computation 
$\Phi(t, x, y)$ defined in $A$, consider the area integral

$$
\int_{A} \frac{\partial \Phi}{\partial y} d A=\int_{a}^{b}\left(\Phi\left(t, x, f_{2}(x)\right)-\Phi\left(t, x, f_{1}(x)\right)\right) d x=\int_{a}^{b}\left([\Phi]_{C_{2}}-[\Phi]_{C_{1}}\right) d x .
$$

As shown in Fig. 4, a positive contour integration corresponds to a counter-clockwise traversal of $C$. To make the first integral in (27) consistent with this connection, we write

$$
\int_{A} \frac{\partial \Phi}{\partial y} d A=-\int_{b}^{a}[\Phi]_{C_{2}} d x-\int_{a}^{b}[\Phi]_{C_{1}} d x=-\int_{C} \Phi d x=-\int_{C} \Phi \frac{d x}{d s} d s
$$

which combines with $\boldsymbol{n}=n_{x} e_{x}+n_{y} e_{y}$ to yield $\int_{A} \frac{\partial \Phi}{\partial y} d A=\int_{C} \Phi n_{y} d s$. A similar computation gives $\int_{A} \frac{\partial \Phi}{\partial x} d A=\int_{C} \Phi n_{x} d s$. Therefore, we have

$$
\int_{A} \nabla \Phi d A=\int_{C} n \Phi d s
$$

where $\nabla \Phi=\left[\frac{\partial \Phi}{\partial x}, \frac{\partial \Phi}{\partial y}\right]^{T}$. It is of interest to note that the total gradient $\int_{A} \nabla \Phi d A$ of the distributed field $\Phi(t, \boldsymbol{\eta})$ over the region $A$ is completely determined from the integral $\int_{C} \boldsymbol{n} \Phi d s$ carried out on the boundary $C$ only. From (29), we can calculate the gradient average of $\Phi(t, \boldsymbol{\eta})$ over the region $A$ as

$$
\bar{\nabla} \Phi=\frac{\int_{C} \boldsymbol{n} \Phi d s}{\Omega_{A}}
$$

where $\Omega_{A}$ is the area of the region $A$. Usually, it is not possible to obtain an explicit result of the integral $\int_{C} \boldsymbol{n} \Phi d s$ because the distributed field $\Phi$ is unknown. Hence, we approximate this integral from measurement $\Phi\left(t, \boldsymbol{q}_{i}\right)$ at the time $t$ and the location $\left(\boldsymbol{q}_{i}\right)$ by each agent $i$, and approximate the area $\Omega_{A}$. We assume that the formation shape is a convex polygon whose vertices are at $\boldsymbol{q}_{i}$. The steps to calculate an approximate value of the integral $\int_{C} \boldsymbol{n} \Phi d s$ and the region area $\Omega_{A}$ are as follows:

1) Using a curve fitting method such as Spline or least square to find a best fitted and smooth contour $C(\theta)$, where $\theta$ is the curve parameter, that goes through all vertices at the time $t$;

2) Calculating an approximate value of $\int_{C} \boldsymbol{n} \Phi d s$ and $\Omega_{A}$ as follows:

$$
\int_{C} \boldsymbol{n} \Phi d s \approx \sum_{i=1}^{N} \Phi\left(t, \boldsymbol{q}_{i}\right) \boldsymbol{n}\left(\theta_{i}\right) \Delta_{C i}, \Omega_{A} \approx \frac{1}{2} \sum_{i=1}^{N}\left(x_{i} y_{i+1}-x_{i+1} y_{i}\right),
$$

where $\boldsymbol{q}_{N+1}=\boldsymbol{q}_{1}, \boldsymbol{n}\left(\theta_{i}\right)$ is the unit vector normal to $C(\theta)$ at $\theta_{i}$ corresponding to the position of the vertex $\boldsymbol{q}_{i}$, and $\Delta_{C i}$ is the arc length from the middle point $M_{i-1}$ between $\boldsymbol{q}_{i-1}$ and $\boldsymbol{q}_{i}$ an the middle point $M_{i+1}$ between $\boldsymbol{q}_{i}$ and $\boldsymbol{q}_{i+1}$, see Fig.5.

For a special case where the formation shape is a regular simple polygon, which has the center at $\boldsymbol{q}_{\text {od }}$ and the vertices at $\boldsymbol{q}_{i}, i \in \mathbb{N}$, and that the contour $C$ goes through all the vertices at the time $t$. Moreover, the unit vector $\boldsymbol{n}$ normal to the contour $C$ at $\boldsymbol{q}_{i}$ is in the direction from $\boldsymbol{q}_{o d}$ to $\boldsymbol{q}_{i}$ at the time $t$. The the integral $\int_{C} \boldsymbol{n} \Phi d s$ and the region area $\Omega_{A}$ can be approximated as

$$
\begin{aligned}
& \int_{C} \boldsymbol{n} \Phi d s \approx \sum_{i=1}^{N} \Phi\left(t, \boldsymbol{q}_{i}\right) \frac{\boldsymbol{q}_{i}-\boldsymbol{q}_{o d}}{\left\|\boldsymbol{q}_{i}-\boldsymbol{q}_{o d}\right\|}\left\|\frac{\boldsymbol{q}_{i+1}-\boldsymbol{q}_{i-1}}{2}\right\|, \\
& \Omega_{A} \approx \frac{1}{2} \sum_{i=1}^{N}\left|\operatorname{det}\left(\left[\boldsymbol{q}_{i}, \boldsymbol{q}_{i+1}\right]\right)\right|,
\end{aligned}
$$

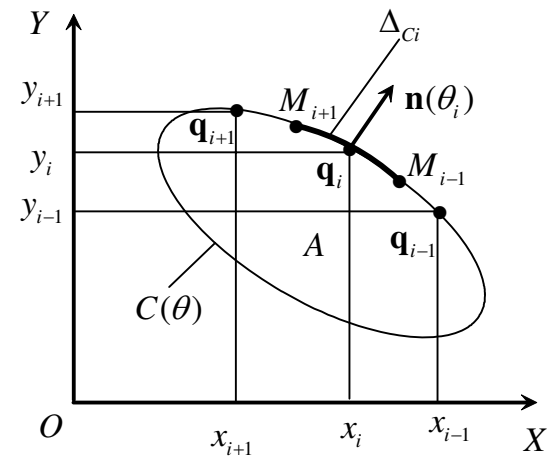

Figure 5: Coordinates for gradient average calculation.

with $\boldsymbol{q}_{N+1}=\boldsymbol{q}_{1}$ and $\boldsymbol{q}_{-1}=\boldsymbol{q}_{N}$, and $\operatorname{det}\left(\left[\boldsymbol{q}_{i}, \boldsymbol{q}_{i+1}\right]\right)$ is the determinant of the matrix $\left[\boldsymbol{q}_{i+1}, \boldsymbol{q}_{i}\right]$. 


\section{Simulation results}

In this section, we a problem of gradient climbing by our proposed formation controller using a group of $N=6$ identical agents. Each agent $i$ has a physical safety radius $\underline{R}_{i}=0.5$ and a communication radius $\bar{R}_{i}=10$. The control design parameters are taken as $k=4, \delta_{i j}=0.5$, $\delta_{i j}^{v}=0.75, c_{i j}=1$, and the bounded function $\psi($.$) taken \operatorname{as} \arctan ($.$) .$

The desired formation shape specification vectors $\boldsymbol{l}_{i d}^{*}$ are chosen as $\boldsymbol{l}_{i d}^{*}=R_{f}\left[\cos \left(\frac{2(i-1) \pi}{N}\right)\right.$, $\left.\sin \left(\frac{2(i-1) \pi}{N}\right)\right]^{T}$ with $R_{f}=3$, and the gain $\boldsymbol{K}_{1 v}=\operatorname{diag}(2.5,2.5)$. This choice of $\boldsymbol{l}_{i d}^{*}$ means that the desired formation configuration is a polygon whose vertices uniformly distribute on a circle centered on the common reference trajectory and with a radius $R_{f}$. The initial conditions are $\boldsymbol{l}_{i d}(0)=\boldsymbol{l}_{i d}^{*}, \boldsymbol{q}_{o d}(0)=\left[\begin{array}{ll}0 & 0\end{array}\right]^{T}$, and $\boldsymbol{q}_{i}(0)=R_{f}\left[\cos \left(\frac{2(i-1) \pi}{N}+\pi\right), \sin \left(\frac{2(i-1) \pi}{N}+\pi\right)\right]^{T}$. These particular initial $\boldsymbol{q}_{i}(0)$ were chosen to illustrate the collision avoidance capability of our proposed formation control system as all the agents have to across the center of the desired formation shape to track their desired reference trajectories. The distributed environment $\Phi(t, x, y)$ is taken as $\Phi(t, x, y)=\mathrm{e}^{-\frac{(x-15)^{2}+(y-15)^{2}}{150}}$, which has a global maximum value at $(x=15, y=15)$.

We set $\boldsymbol{K}_{2 v}=\operatorname{diag}(1.5,1.5)$ to improve the gradient climbing, i.e., the desired formation shape is adapted to the distributed field. Simulation results are plotted in Figure 6. From these figures, it is seen that our proposed formation is able to achieve the objective of both formation control and gradient climbing. The control inputs $\boldsymbol{u}_{i}$, see sub-figure $6 \mathrm{D}$, force the agents to move in such a way that collision between the agents is avoided and that communication between the agents is preserved, see sub-figure $6 \mathrm{~A}$ where trajectories of the agents are plotted in XYplane. These sub-figures also show that our proposed formation control performs the gradient climbing mission very well in the sense that the center of the formation shape, see the polygon of which vertices are the agents, converges to the global maximum location of the function $\Phi(t, x, y)$. Collision avoidance and communication preserving are also confirmed in sub-figure $6 \mathrm{C}$, where the distances $\|\boldsymbol{q}\|_{1 i}$ between the agent 1 and other agents in the group are plotted. These distances are within the range of $(1,10)$ since $\underline{R}_{i}+\underline{R}_{j}=1$ and $\min \left(\bar{R}_{i}, \bar{R}_{j}\right)=10$.
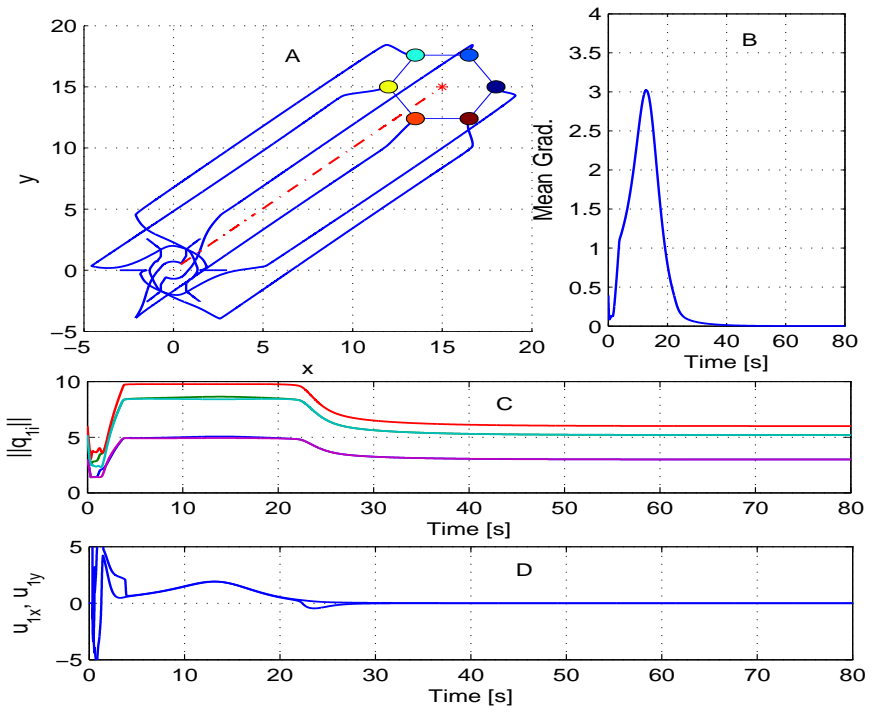

Figure 6: Simulation results with formation shape adaptation. 


\section{Conclusions}

A constructive method has been proposed to design smooth and bounded cooperative controllers for a group of $N$ mobile agents with limited communication to perform a desired formation. Novel potential functions encoding desired formation mission tasks with smooth step functions embedded in were constructed to design the controllers that guaranteed all equilibrium (critical) sets, except for the desired set in formation, are unstable. The proposed formation control system is applied to solve a gradient climbing problem. An extension of the proposed formation control design in this paper and those controllers designed for single underactuated ships in [17] to provide a formation control system for a group of underactuated ships is under consideration.

\section{Proof of Theorem 6}

Proof of no collisions, connectivity preserving, and forward completeness of the closed loop system: It is seen from (23) that $\dot{\varphi} \leq 0$. Integrating $\dot{\varphi} \leq 0$ from $t_{0}$ to $t$ and using the definition of $\varphi$ in (9) with its components defined in (10) and (11) results in

$$
\varphi(t) \leq \varphi\left(t_{0}\right),
$$

where $\varphi\left(t_{0}\right)=\sum_{i=1}^{N}\left(\gamma_{i}\left(t_{0}\right)+\frac{1}{2} \sum_{j \in \mathbb{N}_{i}} \beta_{i j}\left(t_{0}\right)\right)$ and $\varphi(t)=\sum_{i=1}^{N}\left(\gamma_{i}(t)+\frac{1}{2} \sum_{j \in \mathbb{N}_{i}} \beta_{i j}(t)\right)$, for all $t \geq t_{0} \geq 0$. From the condition specified in item 4) of Assumption 3, and properties of $\beta_{i j}$, we have the right hand side of (33) is bounded by a positive finite constant depending on the initial conditions. Boundedness of the right hand side of (33) implies that the left hand side of (33) must be also bounded. As a result, $\beta_{i j}\left(\left\|\boldsymbol{q}_{i j}\right\|^{2} / 2\right)$ must be smaller than some positive constant depending on the initial conditions for all $t \geq t_{0} \geq 0$. From properties of $\beta_{i j}$, see (12), $\left\|\boldsymbol{q}_{i j}\right\|$, for all $(i, j) \in \mathbb{N}$ and $i \neq j$, must be in the interval $\left(\left(\underline{R}_{i}+\underline{R}_{j}\right), \min \left(\bar{R}_{i}, \bar{R}_{j}\right)\right)$. Hence, there are no collisions between any agents and connectivity between agents is preserved for all $t \geq t_{0} \geq 0$. Boundedness of the left hand side of (33) also implies that of $\left(\boldsymbol{q}_{i}(t)-\boldsymbol{q}_{i d}(t)\right)$ also bounded for all $t \geq t_{0} \geq 0$. Moreover, from (21) we can see that $\left|H_{i}\right| \leq 1$. Therefore, $\left\|\boldsymbol{i}_{i d}(t)\right\| \leq\left\|\boldsymbol{v}_{i d}(t)\right\|$ for all $t \geq t_{0} \geq 0$. Therefore, the closed loop system (24) is forward complete.

Equilibrium set: We will use Lemma 2 in [7] to find the equilibrium set, which the trajectories of the closed loop system (24) converge to. Integrating both sides of (23) yields

$$
\int_{0}^{\infty} \omega(t) d t=\varphi\left(t_{0}\right)-\varphi(\infty) \leq \varphi\left(t_{0}\right)
$$

with $\omega(t):=\sum_{i=1}^{N} \boldsymbol{\Omega}_{i}^{T}(t) \Psi\left(\boldsymbol{\Omega}_{\boldsymbol{i}}(\boldsymbol{t})\right)$, where $\boldsymbol{\Omega}_{i}(t)$ is given in (16), and the function $\boldsymbol{\Psi}\left(\boldsymbol{\Omega}_{i}(t)\right)$ is the bounded vector function of $\boldsymbol{\Omega}_{i}(t)$ with properties listed in (18). Indeed, the function $\omega(t)$ is scalar, nonnegative and differentiable. Now differentiating $\omega(t)$ along the solutions of the closed loop system (24) and using properties of the function $\beta_{i j}$ given in (12) readily show that $\left|\frac{\mathrm{d} \omega(t)}{\mathrm{d} t}\right| \leq M \omega(t)$ with $M$ being a positive constant. Therefore Lemma 2 in [7] results in $\lim _{t \rightarrow \infty} \omega(t)=0$, which implies from the expression of $\omega(t)$ and properties of the bounded vector function $\boldsymbol{\Psi}\left(\boldsymbol{\Omega}_{i}(t)\right)$ in (18) that $\lim _{t \rightarrow \infty} \boldsymbol{\Omega}_{i}(t)=0$. Therefore, from the expression of $\boldsymbol{\Omega}_{i}(t)$ the limit $\lim _{t \rightarrow \infty} \boldsymbol{\Omega}_{i}(t)=0$ given in (16) implies that

$$
\lim _{t \rightarrow \infty} \sum_{i=1}^{N}\left(\boldsymbol{q}_{i}(t)-\boldsymbol{q}_{i d}(t)+\sum_{j \in \mathbb{N}_{i}} \beta_{i j}(t) \boldsymbol{q}_{i j}(t)\right)=0 .
$$

The limit in (35) implies that $\boldsymbol{q}(t)=\left[\boldsymbol{q}_{1}^{T}(t) \boldsymbol{q}_{2}^{T}(t), \ldots, \boldsymbol{q}_{N}^{T}(t)\right]^{T}$ can tend to $\boldsymbol{q}_{d}=\left[\boldsymbol{q}_{1 d}^{T} \boldsymbol{q}_{2 d}^{T}, \ldots, \boldsymbol{q}_{N d}^{T}\right]^{T}$ denoted by the set $\boldsymbol{\Xi}_{d}$, since $\beta_{i j}(t)=0$ at $\boldsymbol{q}_{i}=\boldsymbol{q}_{i d}$ and $\boldsymbol{q}_{j}=\boldsymbol{q}_{j d}$, for all $(i, j) \in \mathbb{N}$ and $i \neq j$ or 
tend to the set $\boldsymbol{q}_{c}=\left[\boldsymbol{q}_{1 c}^{T} \boldsymbol{q}_{2 c}^{T}, \ldots, \boldsymbol{q}_{N c}^{T}\right]^{T}$ denoted by the set $\boldsymbol{\Xi}_{c}$ as the time goes to infinity, i.e., the equilibrium sets can be $\boldsymbol{\Xi}_{d}$ or $\boldsymbol{\Xi}_{c}$. The equilibrium set $\boldsymbol{\Xi}_{c}$ is such that

$$
\left.\boldsymbol{\Omega}\right|_{\boldsymbol{q} \in \boldsymbol{\Xi}_{c}}=\left.\left(\boldsymbol{q}_{i}-\boldsymbol{q}_{i d}+\sum_{j \in \mathbb{N}_{i}} \beta_{i j} \boldsymbol{q}_{i j}\right)\right|_{\boldsymbol{q} \in \boldsymbol{\Xi}_{c}}=0
$$

for all $i \in \mathbb{N}$. Thus, we have already proved that the trajectory $\boldsymbol{q}$ can approach either the desired equilibrium set denoted by $\boldsymbol{\Xi}_{d}$ or the undesired equilibrium set denoted by $\boldsymbol{\Xi}_{c}$ 'almost globally'. The term 'almost globally' refers to the fact that the agents start from a set that includes the condition (7) and that does not coincide at any point in the undesired equilibrium set $\boldsymbol{\Xi}_{c}$. Therefore, we now need to prove that $\boldsymbol{\Xi}_{d}$ is a locally asymptotically stable set and that $\boldsymbol{\Xi}_{c}$ is a locally unstable set. Once this is proved, we can conclude that the trajectory $\boldsymbol{q}$ approaches $\boldsymbol{q}_{d}$ from almost everywhere except for from the set denoted by the condition (7) and the undesired equilibrium set $\boldsymbol{\Xi}_{c}$, which is unstable (to be proved below). To prepare for showing that $\boldsymbol{\Xi}_{d}$ is asymptotically stable and that $\boldsymbol{\Xi}_{c}$ is unstable. We write the first equation of the closed loop system (24) for all $i \in \mathbb{N}$ in a vector form as

$$
\dot{\boldsymbol{q}}=-k \Phi\left(\boldsymbol{q}, \boldsymbol{q}_{d}\right)+\dot{\boldsymbol{q}}_{d}
$$

where $\boldsymbol{\Phi}\left(\boldsymbol{q}, \boldsymbol{q}_{d}\right)=\left[\boldsymbol{\Psi}^{T}\left(\boldsymbol{\Omega}_{1}\right), \ldots, \boldsymbol{\Psi}^{T}\left(\boldsymbol{\Omega}_{N}\right)\right]$. Linearizing (37) around $\boldsymbol{q}_{o}=\left[\boldsymbol{q}_{1 o}^{T}, \ldots, \boldsymbol{q}_{N o}^{T}\right]^{T}$, and letting the set $\boldsymbol{\Xi}_{o}$ contain $\boldsymbol{q}_{o}$ results in

$$
\dot{\boldsymbol{q}}=-\left.k \frac{\partial \boldsymbol{\Phi}\left(\boldsymbol{q}, \boldsymbol{q}_{d}\right)}{\partial \boldsymbol{q}}\right|_{\boldsymbol{q} \in \boldsymbol{\Xi}_{o}}+\dot{\boldsymbol{q}}_{d}
$$

where $\frac{\partial \boldsymbol{\Phi}\left(\boldsymbol{q}, \boldsymbol{q}_{d}\right)}{\partial \boldsymbol{q}}=\left[\Delta_{i j}\right]$ with $\Delta_{i j}=\frac{\partial \boldsymbol{\Psi}\left(\boldsymbol{\Omega}_{i}\right)}{\partial \boldsymbol{\Omega}_{i}} \frac{\partial \boldsymbol{\Omega}_{i}}{\partial \boldsymbol{q}_{j}}$ and

$$
\frac{\partial \boldsymbol{\Omega}_{i}}{\partial \boldsymbol{q}_{i}}=\left(1+\sum_{i \in \mathbb{N}_{i}} \beta_{i j}\right)^{\prime} I_{n}+\sum_{j \in \mathbb{N}_{i}} \beta_{i j} / \boldsymbol{q}_{i j} \boldsymbol{q}_{i j}^{T}, \frac{\partial \boldsymbol{\Omega}_{i}}{\partial \boldsymbol{q}_{j}}=-\beta_{i j} I_{n \times n}-\beta_{i j} / \boldsymbol{q}_{i j} \boldsymbol{q}_{i j}^{T},
$$

for all $(i, j) \in \mathbb{N}$. Let $\mathbb{N}^{*}$ be the set of the agents such that if the agents $i$ and $j$ belong to the set $\mathbb{N}^{*}$ then $\left\|\boldsymbol{q}_{i j}\right\| \in\left(\left(\underline{R}_{i}+\underline{R}_{j}\right), \min \left(\bar{R}_{i}, \bar{R}_{j}\right)\right.$. Next we will show that the equilibrium set $\boldsymbol{\Xi}_{d}$ is asymptotically stable and that the equilibrium set $\boldsymbol{\Xi}_{c}$ is unstable.

Proof of $\boldsymbol{\Xi}_{d}$ being asymptotically stable: As mentioned above, to prove that the equilibrium set $\boldsymbol{\Xi}_{d}$ is asymptotically stable, we just need to show that $\boldsymbol{\Xi}_{d}$ is locally asymptotically stable. Letting $\boldsymbol{\Xi}_{o}$ be $\boldsymbol{\Xi}_{d}$ in (38), we obtain

$$
\dot{\boldsymbol{q}}=-k\left(\boldsymbol{q}-\boldsymbol{q}_{d}\right)+\dot{\boldsymbol{q}}_{d},
$$

where we have used the fact that $\left.\beta_{i j}\right|_{\boldsymbol{q} \in \boldsymbol{\Xi}_{d}}=0$ and $\left.\beta_{i j} \prime \prime\right|_{\boldsymbol{q} \in \boldsymbol{\Xi}_{d}}=0$, see Property 1) of the function $\beta_{i j}$ in (12). Local asymptotic stability of the equilibrium set $\boldsymbol{\Xi}_{\boldsymbol{d}}$ follows from (40) since the first time derivative of the function $V_{d}=\frac{1}{2}\left\|\boldsymbol{q}-\boldsymbol{q}_{d}\right\|^{2}$ along the solutions of (40) satisfies $\dot{V}_{d}=-2 k V_{d}$.

Proof of $\boldsymbol{\Xi}_{c}$ being asymptotically stable: Let us define

$$
\begin{aligned}
& \overline{\boldsymbol{q}}=\left[\boldsymbol{q}_{12}^{T}, \ldots, \boldsymbol{q}_{1 N}^{T} \boldsymbol{q}_{23}^{T}, \ldots, \boldsymbol{q}_{2 N}^{T}, \ldots, \boldsymbol{q}_{N-1, N}^{T}\right]^{T}, \overline{\boldsymbol{q}}_{c}=\left[\boldsymbol{q}_{12 c}^{T}, \ldots, \boldsymbol{q}_{1 N c}^{T} \boldsymbol{q}_{23 c}^{T}, \ldots, \boldsymbol{q}_{2 N c}^{T}, \ldots, \boldsymbol{q}_{N-1, N c}^{T}\right]^{T}, \\
& \beta_{i j c}{ }^{\prime}=\left.\beta_{i j}\right|_{\boldsymbol{q} \in \boldsymbol{\Xi}_{c}}, \beta_{i j c} \prime \prime=\left.\beta_{i j}{ }^{\prime \prime}\right|_{\boldsymbol{q} \in \boldsymbol{\Xi}_{c}}, \boldsymbol{q}_{i j c}=\boldsymbol{q}_{i c}-\boldsymbol{q}_{j c} .
\end{aligned}
$$

With the above definitions, we can see that stability of $\boldsymbol{\Xi}_{c}$ is equivalent to that of $\overline{\boldsymbol{\Xi}}_{c}=\overline{\boldsymbol{q}}_{c}$. Define $\boldsymbol{\Omega}_{i j c}=\boldsymbol{\Omega}_{i c}-\boldsymbol{\Omega}_{j c}, \forall(i, j) \in \mathbb{N}, i \neq j$ where $\boldsymbol{\Omega}_{i c}=\left.\boldsymbol{\Omega}_{i}\right|_{\boldsymbol{q} \in \boldsymbol{\Xi}_{c}}=0$, see (36). Therefore $\boldsymbol{\Omega}_{i j c}=0$. Hence $\sum_{(i, j) \in \mathbb{N}^{*}} \boldsymbol{q}_{i j c}^{T} \boldsymbol{\Omega}_{i j c}=0, i \neq j$, which by using (36) is expanded to

$$
\sum_{(i, j) \in \mathbb{N}^{*}}\left(\boldsymbol{q}_{i j c}^{T}\left(\boldsymbol{q}_{i j c}-\boldsymbol{q}_{i j d}\right)+N \beta_{i j c} \boldsymbol{q}_{i j c}^{T} \boldsymbol{q}_{i j c}\right)=0 \Rightarrow \sum_{(i, j) \in \mathbb{N}^{*}}\left(1+N \beta_{i j c} \prime\right) \boldsymbol{q}_{i j c}^{T} \boldsymbol{q}_{i j c}=\sum_{(i, j) \in \mathbb{N}^{*}} \boldsymbol{q}_{i j c}^{T} \boldsymbol{q}_{i j d}
$$


where $i \neq j$. The sum $\sum_{(i, j) \in \mathbb{N}^{*}} \boldsymbol{q}_{i j c}^{T} \boldsymbol{q}_{i j d}$ is strictly negative since at the point $F$ where $\boldsymbol{q}_{i j}=$ $\boldsymbol{q}_{i j d}, \forall(i, j) \in \mathbb{N}^{*}, i \neq j$ all attractive and repulsive forces are equal to zero while at the point $C$ where $\boldsymbol{q}_{i j}=\boldsymbol{q}_{i j c} \forall(i, j) \in \mathbb{N}^{*}, i \neq j$ the sum of attractive and repulsive forces are equal to zero (but attractive and repulsive forces are nonzero). Therefore the point $O$ where $\boldsymbol{q}_{i j}=0, \forall(i, j) \in$ $\mathbb{N}^{*}, i \neq j$ must locate between the points $F$ and $C$ for all $(i, j) \in \mathbb{N}^{*}, i \neq j$. That is the points $F, O$, and $C$ must be co-linear. Hence, there exists a strictly positive constant $b$ such that $\sum_{(i, j) \in \mathbb{N}^{*}} \boldsymbol{q}_{i j c}^{T} \boldsymbol{q}_{i j d}<-b$, which is substituted into (41) to yield

$$
\sum_{(i, j) \in \mathbb{N}^{*}}\left(1+N \beta_{i j c}\right) \boldsymbol{q}_{i j c}^{T} \boldsymbol{q}_{i j c}<-b, i \neq j
$$

Since $\boldsymbol{q}_{i j c}^{T} \boldsymbol{q}_{i j c}>0, \forall(i, j) \in \mathbb{N}^{*}, i \neq j$, there exists a nonempty set $\mathbb{N}^{* *} \subset \mathbb{N}^{*}$ such that for all $(i, j) \in \mathbb{N}^{* *}, i \neq j,\left(1+N \beta_{i j c^{\prime}}\right)$ is strictly negative, i.e., there exists a strictly positive constant $b^{* *}$ such that $\left(1+N \beta_{i j c}\right)<-b^{* *}, \forall(i, j) \in \mathbb{N}^{* *}, i \neq j$.

We now define a subspace $\boldsymbol{\Upsilon}$ as $\boldsymbol{\Upsilon}:=\left(\boldsymbol{q}_{i j}-\boldsymbol{q}_{i j c}=0, \forall(i, j) \in \mathbb{N} \backslash \mathbb{N}^{* *}\right) \cap\left(\boldsymbol{q}_{i j c}^{T}\left(\boldsymbol{q}_{i j}-\boldsymbol{q}_{i j c}\right)=\right.$ $\left.0, \forall(i, j) \in \mathbb{N}^{*}, i \neq j\right)$. In the subspace $\boldsymbol{\Upsilon}$, we have

$$
\bar{V}_{c}=\frac{1}{2} \sum_{(i, j) \in \mathbb{N}^{* *}}\left\|\boldsymbol{q}_{i j}-\boldsymbol{q}_{i j c}\right\|^{2}, \quad \dot{\bar{V}}_{c}=-k \sum_{(i, j) \in \mathbb{N}^{* *}}\left(1+N \beta_{i j c}\right)\left\|\boldsymbol{q}_{i j}-\boldsymbol{q}_{i j c}\right\|^{2} \geq 2 k b^{* *} \bar{V}_{c}
$$

where we have used $\left(1+N \beta_{i j c^{\prime}}\right)<-b^{* *}, \forall(i, j) \in \mathbb{N}^{* *}, i \neq j$. Since the set $\mathbb{N}^{* *}$ is nonempty, (43) implies that the equilibrium set $\overline{\boldsymbol{\Xi}}_{c}$ is unstable by Chetaev's Theorem (Theorem 4.3 in [15]). This implies the desired result that the equilibrium set $\boldsymbol{\Xi}_{c}$ is unstable. We can further explore instability of the equilibrium set $\boldsymbol{\Xi}_{c}$ based on (43) as follows. From (43), we have

$$
\sum_{(i, j) \in \mathbb{N}^{* *}}\left\|\boldsymbol{q}_{i j}(t)-\boldsymbol{q}_{i j c}\right\| \geq \sum_{(i, j) \in \mathbb{N}^{* *}}\left\|\boldsymbol{q}_{i j}\left(t_{0}\right)-\boldsymbol{q}_{i j c}\right\| e^{k b^{* *}\left(t-t_{0}\right)}, i \neq j, t \geq t_{0} \geq 0 .
$$

Now assume that the equilibrium set $\boldsymbol{\Xi}_{c}$ is stable, i.e., $\lim _{t \rightarrow \infty}\left\|\boldsymbol{q}_{i}(t)-\boldsymbol{q}_{i c}\right\|=d_{i}, \forall i \in \mathbb{N}$ with $d_{i}$ a nonnegative constant. Note that $\mathbb{N}^{* *} \subset \mathbb{N}$, we have $\lim _{t \rightarrow \infty}\left\|\boldsymbol{q}_{i}(t)-\boldsymbol{q}_{i c}\right\|=d_{i}, \forall i \in \mathbb{N}^{* *}$, which implies that $\lim _{t \rightarrow \infty} \sum_{(i, j) \in \mathbb{N}^{* *}}\left\|\boldsymbol{q}_{i j}(t)-\boldsymbol{q}_{i j c}\right\|=d^{* *}, \forall(i, j) \in \mathbb{N}^{* *}, i \neq j$ with $d^{* *}$ a nonnegative constant, since $\boldsymbol{q}_{i j}=\boldsymbol{q}_{i}-\boldsymbol{q}_{j}$ and $\boldsymbol{q}_{i j c}=\boldsymbol{q}_{i c}-\boldsymbol{q}_{j c}$. This contradicts (44) for the case $\sum_{(i, j) \in \mathbb{N}^{* *}}\left\|\boldsymbol{q}_{i j}\left(t_{0}\right)-\boldsymbol{q}_{i j c}\right\| \neq 0$, since the right hand side of (44) is divergent (so does the left hand side). For the case $\sum_{(i, j) \in \mathbb{N}^{* *}}\left\|\boldsymbol{q}_{i j}\left(t_{0}\right)-\boldsymbol{q}_{i j c}\right\|=0$, there would be no contradiction. However this case is never observed in practice since the ever-present physical noise would cause $\left\|\boldsymbol{q}_{i j}\left(t^{*}\right)-\boldsymbol{q}_{i j c}\right\|$ for some $(i, j) \in \mathbb{N}^{* *}, i \neq j$ to be different from 0 at the time $t^{*} \geq t_{0}$. Proof of Theorem 6 is completed.

\section{Bibliography}

[1] A. Das, R. Fierro, V. Kumar, J. Ostrowski, J. Spletzer, and C. Taylor, "A vision based formation control framework," IEEE Transactions on Robotics and Automation, vol. 18, no. 5, pp. 813-825, 2002.

[2] J. Hu and G. Feng, "Distributed tracking control of leader-follower multi-agent systems under noisy measurement," Automatica, vol. 46, no. 8, pp. 1382-1387, 2010.

[3] M. Egerstedt and X. Hu, "Formation constrained multiagent control," IEEE Transactions on Robotics and Austomation, vol. 17, no. 6, pp. 947-951, 2001. 
[4] T. Balch and R. C. Arkin, "Behavior-based formation control for multirobot teams," IEEE Transactions on Robotics and Automation, vol. 14, no. 6, pp. 926-939, 1998.

[5] R. T. Jonathan, R. W. Beard, and B. Young, "A decentralized approach to formation maneuvers," IEEE Transactions on Robotics and Automation, vol. 19, no. 6, pp. 933-941, 2003.

[6] H. G. Tanner and A. Kumar, "Towards decentralization of multi-robot navigation functions," in Proceedings of the 2005 IEEE International Conference on Robotics and Automation, (Barcelona, Spain), pp. 4132-4137, 2005.

[7] K. D. Do, "Bounded controllers for formation stabilization of mobile agents with limited sensing ranges," IEEE Transactions on Automatic Control, vol. 52, no. 3, pp. 569-576, 2007.

[8] E. Rimon and D. E. Koditschek, "Exact robot navigation using artificial potential functions," IEEE Trans. Robot. and Automat., vol. 8, no. 5, pp. 501-518, 1992.

[9] D. M. Stipanovic, G. Inalhan, R. Teo, and C. J. Tomlin, "Decentralized overlapping control of a formation of unmanned aerial vehicles," Automatica, vol. 40, no. 8, pp. 1285-1296, 2004.

[10] R. Olfati-Saber, "Flocking for multi-agent dynamic systems: algorithms and theory," IEEE Transactions on Automatic Control, vol. 51, no. 3, pp. 401-420, 2006.

[11] M. Ji and M. Egerstedt, "Distributed coordination control of multi-agent systems while preserving connectedness," IEEE Transactions on Robotics, vol. 23, no. 4, pp. 693-703, 2007.

[12] M. M. Zavlanos and G. J. Pappas, "Potential fields for maintaining connectivity of mobile networks," IEEE Transactions on Robotics, vol. 23, no. 4, pp. 812-816, 2007.

[13] D. V. Dimarogonas and K. J. Kyriakopoulos, "Connectedness preserving distributed swarm aggregation for multiple kinematic robots," IEEE Transactions on Robotics, vol. 24, no. 5, pp. 1213-1222, 2008.

[14] K. D. Do, "Output-feedback formation tracking control of unicycle-type mobile robots with limited sensing ranges," Robotics and Autonomous Systems, vol. 57, pp. 34-47, 2009.

[15] H. Khalil, Nonlinear Systems. Prentice Hall, 2002.

[16] M. Krstic, I. Kanellakopoulos, and P. Kokotovic, Nonlinear and Adaptive Control Design. New York: Wiley, 1995.

[17] K. D. Do and J. Pan, Control of Ships and Underwater Vehicles: Design for Underactuated and Nonlinear Marine Systems. Springer, 2009. 